\title{
EFFECT OF PANDEMIC ON STOCK PRICE BEHAVIOUR WITH SPECIAL REFERENCE TO S\&P BSE HEALTHCARE INDEX
}

\author{
Sucheesmita Dash ${ }^{1}$, \\ ${ }^{1}$ M.Com. 2nd Year, Department of Commerce, Ravenshaw University, Cuttack, \\ Prof. (Dr.) Sanjay Kumar Satapathy ${ }^{2}$ \\ ${ }_{2}^{2}$ Dean, School Of Commerce, Department of Commerce, Ravenshaw University, Cuttack,
}

Article DOI: https://doi.org/10.36713/epra5409

\begin{abstract}
The outburst of pandemic has caused widespread disorder in the country and the stock market is not also free from its grip. Despite the decrement in the performance of sectoral indices, the pharma sector has outperformed in both the NSE and BSE stock exchanges. The present study focuses on the market behavior of S\&P BSE Healthcare Index and its top ten constituent companies' pre and post pandemic outbreak. The market behavior is measured in terms of the closing price of the index and its constituents which is being studied by using $t$-test. The results of the study indicated that there is no deviation in the performance of $S \& P$ BSE Healthcare Index after the outburst of pandemic. While eight of its constituent companies showed deviation in their performance post pandemic outbreak. Out of which most of the companies showed positive deviation post pandemic outburst. Therefore, it would be beneficial for the investors to invest in the pharma sector stocks amid the national crisis.
\end{abstract}

KEYWORDS: Pandemic, Epidemic, Risk, Pharma sector.

\section{INTRODUCTION}

An epidemic may be defined as the occurrence of large scale disease in a community or region as compared to the normal expected disease. An epidemic that covers a wide range of area whereby international boundaries are crossed and illness is caused to people in large numbers is called a pandemic. The former one (i.e., epidemic) affects a large community or region within the national boundaries and the later one (i.e., pandemic) affects people beyond the national boundaries, i.e., crossing the international boundaries. Pandemics is generally an infectious disease that spreads over a large geographical dimension that increases the chances of people being affected by that disease and increases the mortality rate. This leads to large scale of social, economic and political problems. There had been notable pandemic outbreaks in the past such as Spanish Flu in 1920, Cholera outbreak in 1820, Plague in 1720, etc.

Pandemic occurs due to a number of factors such as population explosion, increased urbanization, increased amount of animal protein in the diet, increased capacity of travel and tourism connecting a large number of people across the world, climate change, global warming, increased interface between human and animal has increased both spark and spread risk of pandemics. It is becoming difficult to detect and contain a pandemic spread with such a large number of population worldwide with increasing number of travel and trade.

One such pandemic is coronavirus which affected India on $30^{\text {th }}$ January, 2020. This infectious disease outbreak was declared as a pandemic due its large geographical coverage crossing international boundaries. Amid the pandemic outbreak the government has declared a complete lockdown similar to a curfew in all the states and union territories. From the past trends, it is evident that there were many pandemic outbreaks and there will an increase in the number in the future as well due to the increasing count of viral infections/ diseases from animals. There are two types of risks associated with a pandemic: 
- $\quad$ Spark Risk: As the name suggests, it means where a pandemic is very likely to originate or arise.

- $\quad$ Spread Risk: As the name suggests, it means how likely it is that a pandemic will spread among the large population of an area.

A lot of consequences come hand in hand with the pandemic outbreak. Some of the consequences caused due to pandemic outbreak include health impact, economic impact and social and political impacts. Pandemic outbreak cause a number of health problems. Pandemics can cause widespread and significant morbidity and mortality over a wide dimension of population. The worse affected are the LMICs. Moreover, when a pandemic hits, it is not necessary that a country has fully prepared (particularly sufficient numbers of) healthcare units to serve the infected patients which makes it difficult to provide even necessary medical services to the infected ones leading to increased mortality rates in the country. The outbreak of a pandemic worsely affects the economic conditions of a country. It leads to short-term fiscal shortage and long-term damage to economic growth in terms of GDP. In case of Pandemic, other than the essential commodities' production and distribution, all other production and distribution facilities remain closed that badly affects the growth of an economy and put it in a setback situation. It is evident from the past that epidemic and pandemic have caused great political and social consequences. This has led to great social tension and discrimination. People are being isolated, their lives are in havoc, and there arises a great number of political unrest due to pandemic outbreaks.

The pandemic outbreak has caused chaos everywhere and the stock market is also not free from its clutches. At one side, when the Global equity market most Asian and European markets are being haunted by the pandemic outbreak and have registered huge losses in the economic breakdown. Indian stock market on the other side is experiencing a roller coaster ride. It is evident as Sensex ended $1,627.73$ points higher at 29,915.56 while Nifty was up 482 points at $8,745.45$ (as per $21^{\text {st }}$ March, 2020 Ace Equity).

Amid the uncertainty among the investors due to the pandemic outbreak and fall in the market, the Pharma sector outperformed the other markets both in BSE and NSE stock exchanges. The increase in the healthcare stock prices is on the back of the global trend. The sudden rise in the share prices of pharmaceutical companies can be traced to the increased requirements for generics and branded generics. It has recently led to investors rushing for pharmaceutical stocks due to rise in demand for generics and branded generics resulting in shortages and over-pricing or increasing price of the drugs. Furthermore, the ban on API (Active Pharmaceutical Ingredients) and export ban on paracetamols, antibiotics and vitamins has alarmed the investors over the impact of the pandemic and the fight against the outbreak.

\section{REVIEW OF LITERATURE}

Many researchers have studied the impact of pandemic outbreak on the economy of the country. It is quite natural that any pandemic outbreak will lead to many consequences. The results of some of the major studies are outlined below. It can be noted that many researchers had studied the consequences of pandemic outbreak on the GDP or the economy of the country. But yet there are no studies that are being conducted on the impact of pandemic outbreak on the performance of healthcare indices. Hence, the study attempts to fill the gap by examining the performance of S\&P BSE Healthcare Index and its top 10 constituents. The results of the present study would contribute to the current stock of knowledge and would help to further extent the literature on the theme under study. Meltzer et al. (1999) state the future possible effects of next pandemic influenza in United States and studied how the intervention in terms of vaccines can impact the economy. They further state that vaccinating $60 \%$ of the population can increase the economic returns and decrease the loss by many folds but it is very difficult job to vaccinate people within the given time if vaccines are required in two doses. Brainerd (2003) state that the death toll during the influenza epidemic (1918-19) was disproportionately more than the death during World War-I. Epidemic followed a W-shaped death as compared to the normal or ordinary U-shape. The increased mortality rate had a positive effect on the economic growth in United States. Overby et al. (2004) states that SARS emerged in China in November, 2002 and spread to 26 countries. The SARS epidemic caused severe economic crisis in Southeast Asia. SARS affected the investment in China as the investors considered the country as riskier for investment. Moreover, the SARS affected the supply chains making the business in China unreliable.

Bloom et al. (2005) states that Avian Flu has a potential impact on the development of a nation. No one actually predict the consequences of a new Flu Pandemic. They further state that there will be worse consequences if the pandemic continues for a quite long time. Public policy can play a great role in determining the economic consequences. Burns et al. (2006) state a pandemic can spread in no time not only in the home country but also can spread to other countries and regions. Thus, combined efforts are essential to develop strategies and plans in order to combat the pandemic. Research and development at global level is essential for overcoming the pandemic. Garrett (2007) states that the 1918 influenza pandemic had a relatively short term impact on business. Many businesses, specifically those engaged in services and entertainment suffered huge amount of losses on the other hand the businesses 
that were engaged in healthcare products experienced an increase in their revenues. Enemark (2009) states that the microbial threat or pandemic has increased the interdependence of the states. The solution lies in international cooperation by sharing their health information, healthcare units and increasing as well as expanding the supply of vaccines for influenza.

Doshi (2011) states that the definition of novel H1N1 influenza. He states that despite 10 years of pandemic preparedness there is no proper definition of a 'Pandemic Influenza' given by WHO. The underlying assumptions of the very nature of this infectious disease is unknown due to the lack of a proper definition. Karlsson et al. (2014) state the impact of 1918 Spanish Influenza on the economic performance of Sweden. They further state how the influenza affected the earnings or livelihood, returns on the capital and the poverty in the country. In this study, they found that there was negative returns on capital, increase in poverty rates and but there was no detectable impact on the earnings which goes against the previous studies conducted. Sahu et al. (2017) state the spread of pandemic over Central India focusing mainly on MP state which the worst affected by the 2009 pandemic outbreak. The study emphasized that the number of adults were more than the number of children affected by the disease. Moreover, among the adults, the number of women were more than the number of men affected by the disease. The reason can be the lack of proper hygiene and medical facilities the women.

Holmberg et al. (2018) states that the pandemic preparedness programme of eight European countries published after 2009. They emphasized that the main aim of pandemic preparedness plan is to create awareness among the public about the pandemic and the precautions that are to be taken to avoid it. Different countries have different pandemic preparedness plans but to combat the pandemic outbreak, they internationally coordinate. Fan et al. (2018) state the need for more investment in preparation against epidemics and pandemics. They further state that earlier the need for investment was felt only on the basis of industrial and macroeconomic losses but in this study, they also considered the value of lives lost due to the pandemic outbreak. This increased the loss incurred to many folds as with the pandemic outbreak, the mortality rate also increases subsequently. Kostova et al. (2019) state the role of the 2014 West Africa Ebola outbreak in U.S. merchandise exports to Ebola affected countries and U.S. jobs supported by these exports. Epidemics could disrupt travel and transportation logistics through curfews, travel restrictions, and market closures and could lower foreign direct investment, shift the internal resource allocations of affected countries, and affect currency values. Guimbeau et al. (2019) state that the effects of 1918 Influenza Pandemic over Sao Paulo, Brazil.
They emphasized that the pandemic outbreak had a significant impact on the mortality among infants, sex ratios at birth and productivity in agriculture in 1920. It further emphasized that the impact persists in 1940 as well.

Meng et al. (2020) state that infectious control measures should be undertaken to prevent further spread of infectious disease. They also further highlighted that there is high chances of cross risk infections between patients and dental practitioners. Necessary initiatives are to be taken for dental practices \& hospitals in areas that are potentially affected with COVID-19. Delivorias et al. (2020) states that the outbreak of an influenza have serious repercussions for national and regional economies. The pandemic/ epidemic outbreak have serious impacts on several sectors such as healthcare, transportation, tourism and agricultural sectors. The first sector that is adversely affected is the healthcare sector as the increase in the number of infected patients there is a surge in the administrative and operational expenditures. McKibbin et al. (2020) have examined the impacts of COVID-19 from seven different scenarios on macro-economic outcomes and financial markets. These study further highlights how investment in public healthcare systems could contain the virus outbreak in less-developed but highly populous economies. Rajagopal (2020) state on account of COVID-19 outbreak, there is a shortage of API (Active Pharmaceutical Ingredients) that is imported from China that would affect the pharma players adversely. Indian pharma sector is heavily depended on China for API as well as other intermediary products for production of various medicines. If the virus is not contained within 9 months, then it would affect the performance of big pharma companies as well. Mahesh (2020) state that despite of supply chain disruptions amid the fall in sectoral indices and sell off in the mutual funds, pharma sector is the least affected one. The aggressive investors can add $5 \%$ of pharma sector in their portfolio.

Somvanshi (2020) state that the traditional way of selling low cost generic drugs is not more a good option to build business in the US market and thus the Indian pharma companies have been trying too hard by adopting various strategies. In this regard, Sun pharma, Cipla, Aurobindo Pharma and various other pharma companies have extended their focus into specialty drugs that are complex to manufacture and requires huge investment in R\&D. Langlois (2020) state the situation now is somehow similar to the situation of 1918 Spanish Flu Influenza in which the market suffered the bottom by the December 1917. If not wrong in 2020 as well if the number of casualties increase there will be a great impact on the stock market. But we can cope with the situation as there is a number of good hospitals, medication as compared to the 1918 Spanish Flu. 


\section{OBJECTIVES}

The present paper has the following objectives:

1. To study the trends of market behaviour of S\&P BSE Healthcare Index and its top 10 constituents pre and post pandemic outbreak.

2. To study effect of pandemic on the market behavior of S\&P BSE Healthcare Index and its top 10 constituents.

\section{RESEARCH METHODOLOGY}

The present study is an ex-post facto and analytical research. Ex-post facto research is one type of descriptive research. This type of research involves analyzing a set of data after the occurrence of a particular event. On the other hand, analytical research involves doing a critical analysis of a present set of data.

\section{Period of the study:}

For the purpose of present study, 50 days before and after the pandemic outbreak has been taken into consideration. For ease, 50 days before the pandemic outbreak is referred to as 'pre-pandemic period' and the 50 days after the pandemic outbreak is referred to as 'post-pandemic period'. These 50 days before and after pandemic outbreak includes the Saturdays, Sundays and public holidays on which the stock exchange remained closed thus, the final number of days taken into consideration for the study is 35 days before and after pandemic outbreak.

\begin{tabular}{|l|l|}
\hline Pre-Pandemic Period & 12.12 .2019 to 30.01 .2020 \\
\hline Post-Pandemic Period & 31.01 .2020 to 22.03 .2020 \\
\hline
\end{tabular}

\section{Sources of Data}

For the present study, secondary data has been used and which has been collected from online website. For the present study, S\&P BSE Healthcare Index and its top 10 constituents companies are being considered. The closing price is being taken as a measure of the market behavior from the official website of BSE, i.e., www.bseindia.com
1. Sun Pharma
2. Dr. Reddy's Laboratories Ltd.
3. Divi's Laboratories Ltd.
4. Cipla Ltd./ India
5. Piramal Enterprises Ltd.

\section{Methods of Analysis}

For the present study, descriptive statistics, trend analysis and paired t-test has been used for analyzing the data and interpreting the results. Graphs have been used to show the trend in performance of the indices.

\section{Hypothesis}

$\mathbf{H}_{\mathbf{o}}$ : There is no significant difference in market behavior of S\&P BSE Healthcare Index and its top 10 constituents pre and post pandemic outbreak.

\section{Sample Size}

For the purpose of the present study, S\&P BSE Healthcare Index and its top 10 constituents are taken into consideration. The top 10 constituents of S\&P BSE Healthcare Index are the following:
6. Apollo Hospitals Enterprise Ltd.
7. Lupin Ltd.
8. Aurobindo Pharma Ltd.
9. Biocon Ltd.
10. Torrent Pharmaceuticals Ltd

$\mathbf{H}_{1}$ : There is significant difference in market behavior of S\&P BSE Healthcare Index and its top 10 constituents pre and post pandemic outbreak.

\section{RESULTS AND DISCUSSION}

This section deals with the trends of the stock prices of sample companies and the Healthcare Index. The trends of stock prices are shown both before and after the pandemic outbreak in India with the help of graphs for each sample separately.

\section{Figure 1: Stock Price Behaviour of S\&P BSE Healthcare Index}

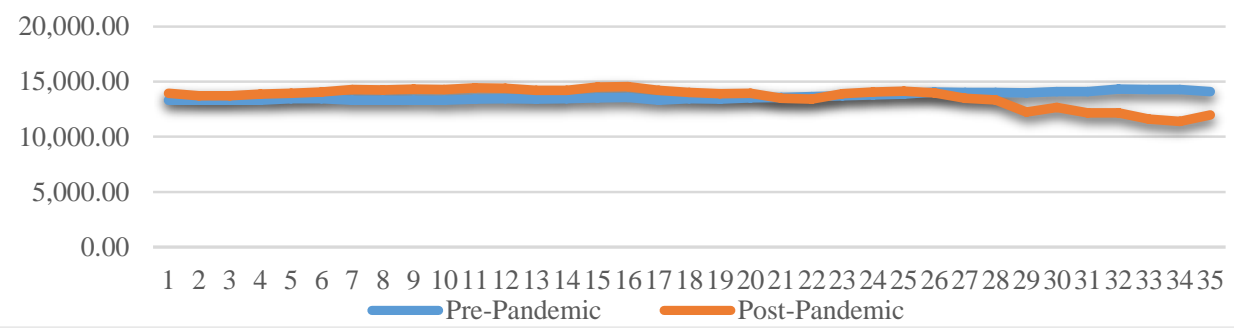


Figure 3 shows the market behavior of Dr. Reddy's Laboratories Ltd. pre and post pandemic outbreak. On the basis of above information about Dr. Reddy's Laboratories Ltd., we can say that there has been a surge in the performance of its stocks after the pandemic outbreak. Thus, it can be said that there has been a positive impact of pandemic outbreak on the market behavior of Dr. Reddy's Laboratories Ltd.
Figure 4 depicts the market behavior of Divi's Laboratories Ltd. pre and post pandemic outbreak. On the basis of the above information, we can say that after the pandemic outbreak there has been a gradual increase in the performance of the Divi's Laboratories ltd. Thus, the outbreak of the pandemic has a positive impact on its market behavior.

Figure 2: Stock Price Behavior of Sun Pharma

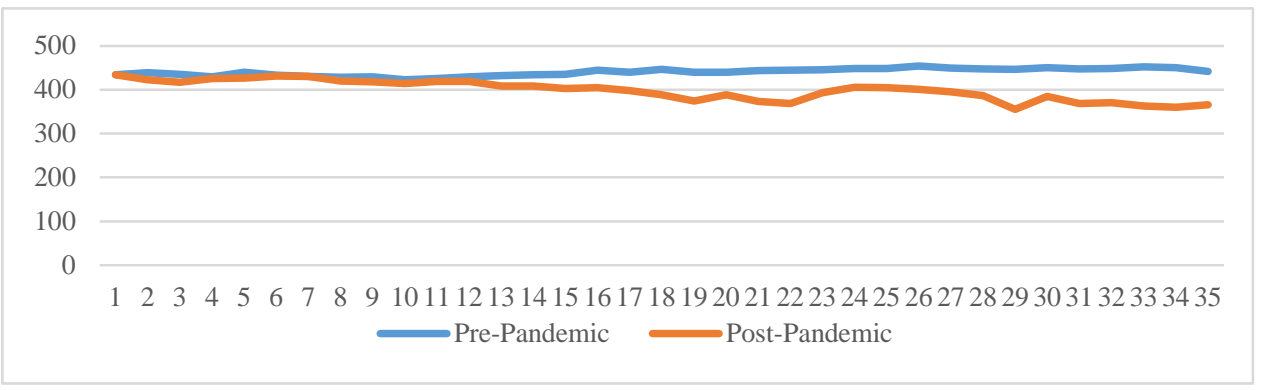

Figure 1 shows the market behavior of the S\&P BSE Healthcare Index pre and post pandemic outbreak. It is very clear from the above graph that there has been a surge in the price of the S\&P BSE Healthcare index stock price after the pandemic outbreak. It may be because of the heavy dependence of public on healthcare and medical services. The price is highest on the $16^{\text {th }}$ day of the post pandemic outbreak with ₹ 14,535 . Figure 2 shows the market behavior of Sun Pharma pre and post pandemic outbreak. We can say on the basis of above information related to Sun Pharma that there is a gradual decrease in its performance after the pandemic outbreak. The performance of the Sun Pharma was better before the pandemic outbreak. So we can say that the pandemic outbreak affected its performance significantly.

Figure 3: Stock Price Behaviour of Dr. Reddy's Laboratories Ltd.

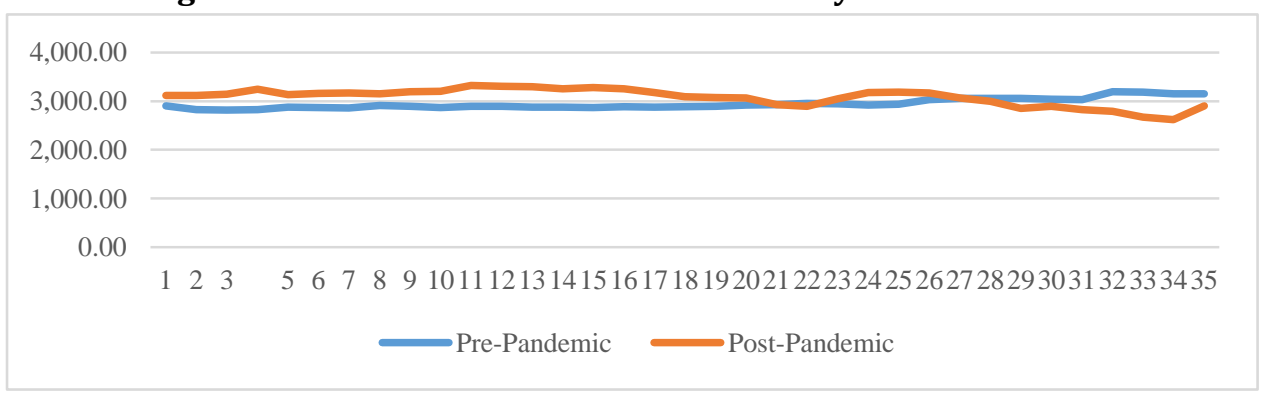

Figure 4: Stock Price Behavior of Divi's Laboratories Ltd.

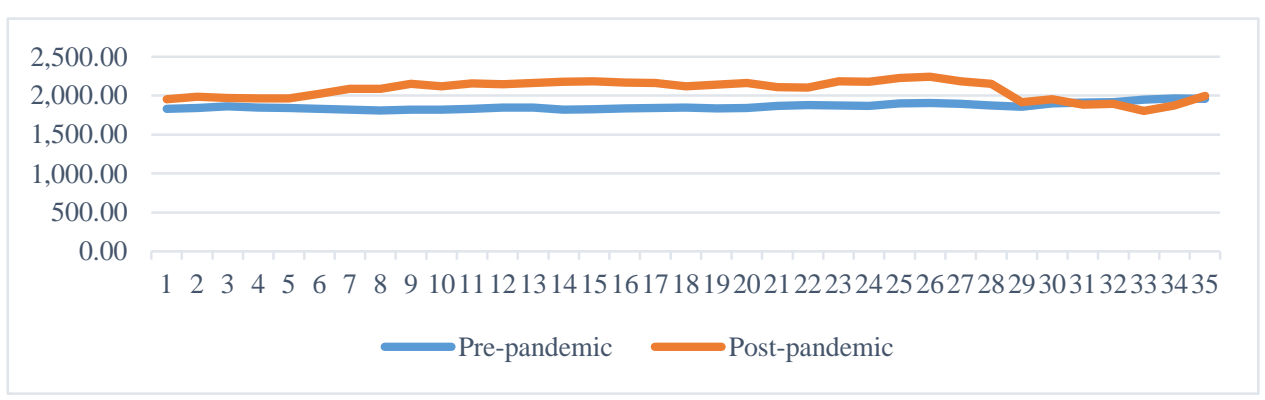


Figure 5: Stock Price Behavior of Cipla Ltd

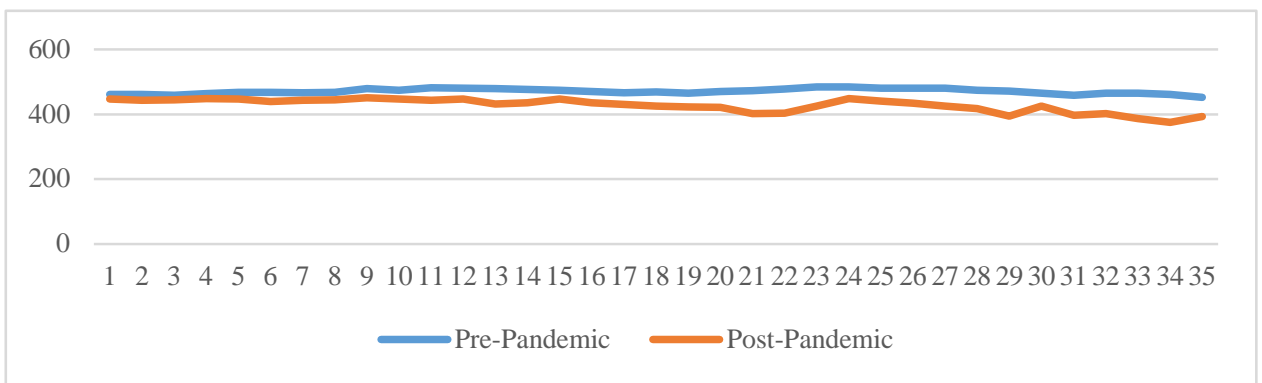

Figure 5 represents the market behavior of Cipla Ltd. India pre and post pandemic outbreak. It is clear from the above information that pandemic outbreak had a negative impact on the market behavior of Cipla Ltd. India as after the pandemic outbreak there has been a constant decline in its performance. Figure 6 represents the market behavior of Piramal Enterprises Ltd. pre and post pandemic outbreak. From the above information we can say that there has been a negative as well as a positive impact after the pandemic outbreak on the Piramal Enterprises Ltd. performance. Gradually, after the pandemic outbreak it declined but suddenly in between it increased and again it declined. No doubt, there was worse than good for the Piramal Enterprises Ltd. Figure 7 represents the market behavior of Apollo Hospital Enterprises Ltd. pre and post pandemic outbreak. From the above information about the market behavior of Apollo Hospitals Enterprise Ltd., we can say that there has been a positive impact of the pandemic outbreak on its performance. As we can see, there has been a great increase in its performance since the pandemic outbreak. Although, it declined after $28^{\text {th }}$ day of the pandemic outbreak.

Figure 6: Stock Price Behavior of Piramal Enterprises Ltd.

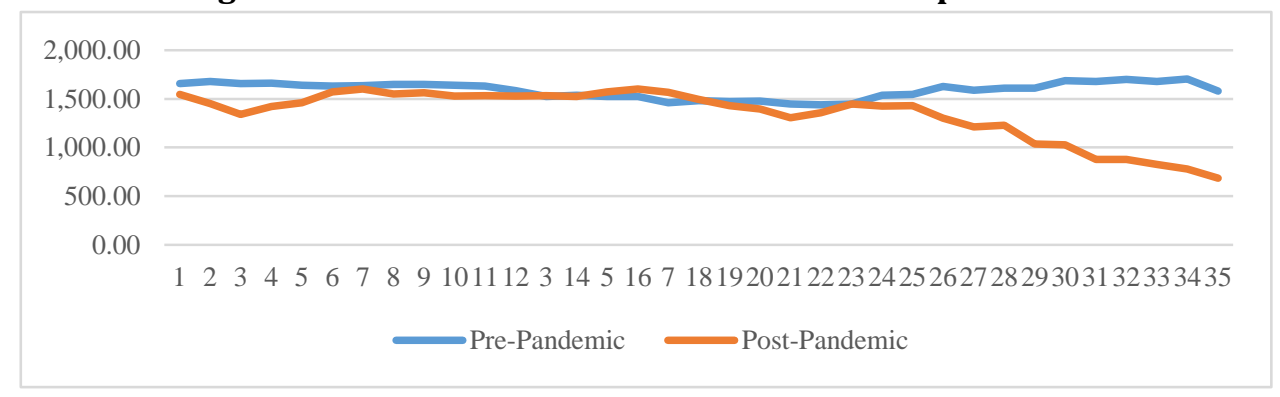

Figure 8 represents the market behavior of Lupin Ltd. pre and post pandemic outbreak. We can say on the basis of above information about the market behavior of Lupin Ltd. that the pandemic outbreak has a negative impact on its performance. There has been a decrease in its performance since the pandemic outbreak.

Figure 7: Stock Price Behavior of Apollo Hospitals Enterprise Ltd.

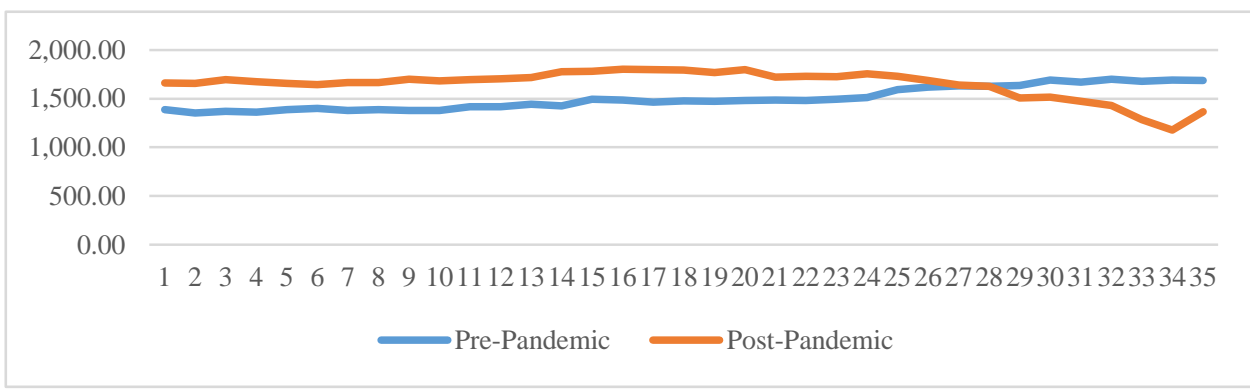


Figure 8: Stock Price Behavior of Lupin Ltd.

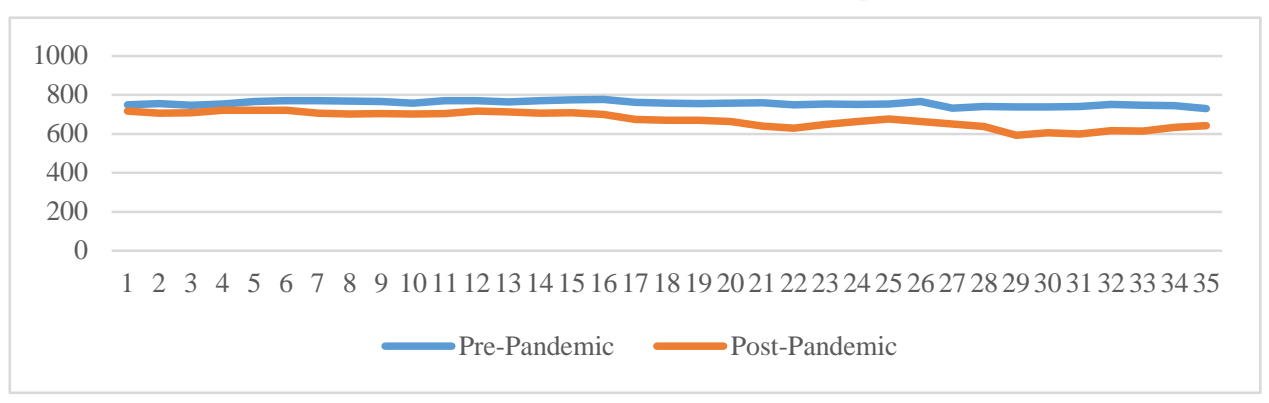

Figure 9: Stock Price Behavior of Aurobindo Pharma Ltd.

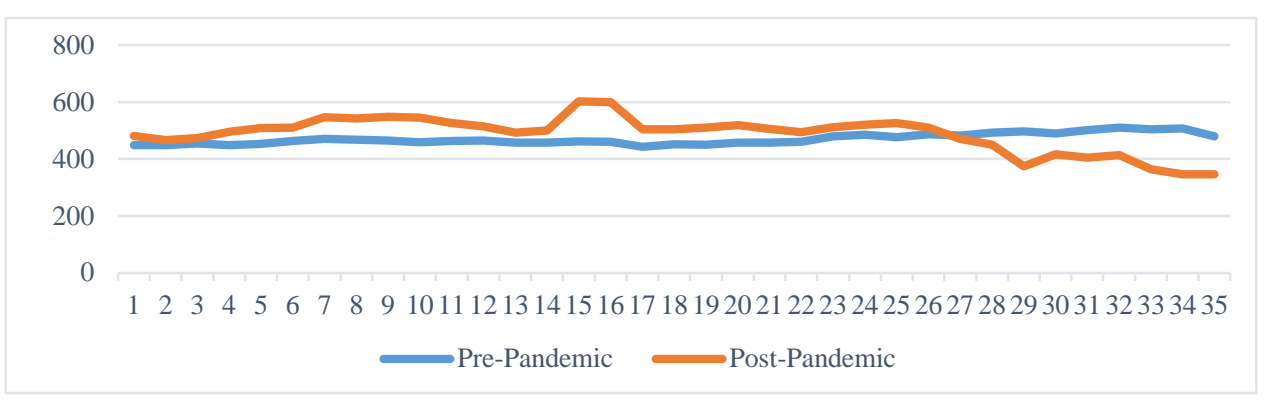

Figure 9 represents the market behavior of Aurobindo Pharma Ltd. pre and post pandemic outbreak. On the basis of above information about the market behavior of Aurobindo Pharma Ltd. pre and post pandemic outbreak, the pandemic outbreak has a positive impact on its performance as there has been a considerable increase in its performance. Figure 10 represents the market behavior of Biocon
Ltd. pre and post pandemic outbreak. On the above information about the market behavior of Biocon Ltd., the pandemic outbreak has a mixed impact on its performance as initially there was a decline in its performance which eventually increased with the passing days. Figure 11 represents the market behavior of Torrent Pharmaceuticals Ltd. pre and post pandemic outbreak. On the basis of above information about the market behavior of Torrent Pharmaceuticals Ltd. pre and post pandemic outbreak, we can say that there is a positive impact of pandemic outbreak on the performance of the company. Since pandemic outbreak its performance has increased considerably.

Figure 10: Stock Price Behavior of Biocon Ltd.

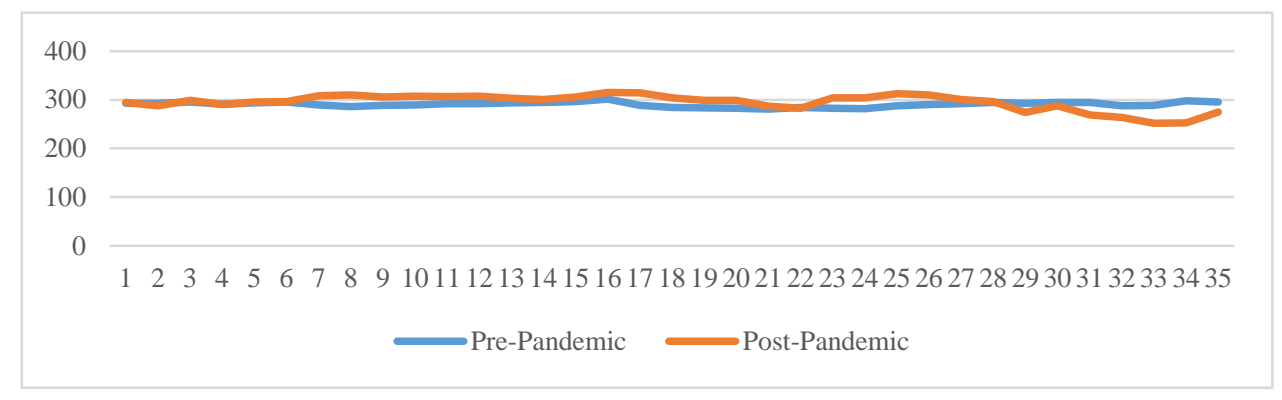


Figure 11: Performance of Torrent Pharmaceuticals Ltd.

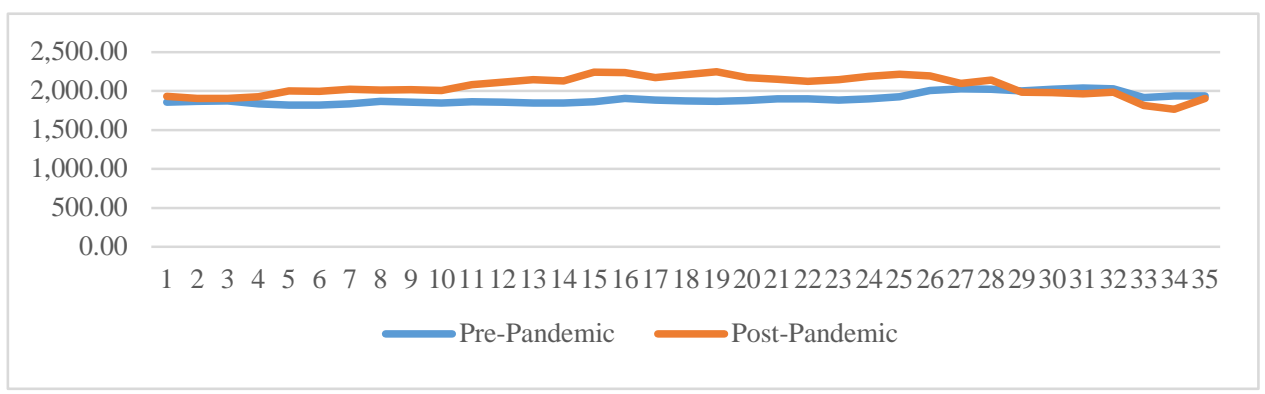

\section{Descriptive Statistics}

Table 1 shows the summarized statistics for the S\&P BSE Healthcare index and its top ten constituents.

Table 1: Descriptive Statistics

\begin{tabular}{|c|c|c|c|c|c|c|c|}
\hline Variable & Mean & SD & Minimum & Median & Maximum & Skewness & Kurtosis \\
\hline S\&PPre & 13668 & 322 & 13319 & 13520 & 14310 & 0.77 & -0.94 \\
\hline S\&PPost & 13629 & 875 & 11397 & 13947 & 14535 & -1.34 & 0.64 \\
\hline SunPre & 440.24 & 8.51 & 422.3 & 440.1 & 454.45 & -0.3 & -0.99 \\
\hline SunPost & 398.51 & 23.12 & 354.85 & 403.25 & 434 & -0.3 & -1.1 \\
\hline ReddyPre & 2947.5 & 105.3 & 2816.3 & 2905.5 & 3188.9 & 1.08 & 0.13 \\
\hline ReddyPost & 3078.2 & 178.7 & 2622.8 & 3134.5 & 3325.7 & -0.92 & 0.24 \\
\hline DiviPre & 1862 & 41.1 & 1806.8 & 1846.3 & 1961.3 & 1.01 & 0.29 \\
\hline DiviPost & 2073.3 & 116.4 & 1805.1 & 2117.3 & 2240.3 & -0.65 & -0.78 \\
\hline CiplaPre & 470.42 & 8.16 & 451.5 & 470 & 484.4 & -0.13 & -0.66 \\
\hline CiplaPost & 427.55 & 20.9 & 374.6 & 433.95 & 450.65 & -0.95 & -0.15 \\
\hline PiramalPre & 1587.8 & 82.6 & 1436.3 & 1611.5 & 1702.3 & -0.46 & -1.11 \\
\hline PiramalPost & 1343.3 & 263.4 & 684.8 & 1429.2 & 1601 & -1.25 & 0.39 \\
\hline ApolloPre & 1501.3 & 115.9 & 1353.6 & 1479.7 & 1697.3 & 0.5 & -1.21 \\
\hline ApolloPost & 1648.2 & 147.7 & 1178.6 & 1688.3 & 1800.2 & -1.67 & 2.6 \\
\hline LupinPre & 756.01 & 12.44 & 728.8 & 756.25 & 776.5 & -0.32 & -0.67 \\
\hline LupinPost & 672.85 & 39.93 & 593.05 & 673.25 & 722.35 & -0.47 & -1.04 \\
\hline AboPre & 470.47 & 18.87 & 443.55 & 464 & 511 & 0.69 & -0.66 \\
\hline AboPost & 487.1 & 63.4 & 345.8 & 504.1 & 602.3 & -0.76 & 0.35 \\
\hline BioconPre & 290.46 & 5.05 & 281.05 & 291.85 & 301.05 & -0.31 & -0.6 \\
\hline BioconPost & 294.82 & 16.6 & 252 & 299.2 & 315 & -1.23 & 0.9 \\
\hline TorrentPre & 1902.2 & 66.5 & 1817.3 & 1878.3 & 2036.9 & 0.94 & -0.36 \\
\hline TorrentPost & 2060.9 & 126.4 & 1767.1 & 2081.1 & 2249.6 & -0.37 & -0.65 \\
\hline
\end{tabular}

It is evident from the summarized result that the average performance of S\&P BSE Healthcare Index is better in pre-pandemic outbreak than in the post pandemic outbreak. But if we focus on the average performance of its top ten constituents we can see that their average performance is better after the pandemic outbreak. The standard deviation is an indication of deviation of returns and it is evident from the above summary that the deviation is more in post pandemic period in S\&P BSE Healthcare Index and its top ten constituents. On the basis of the descriptive statistics we can say that, among the top 10 constituents of S\&P BSE Healthcare Index, most of the pharma sector companies have shown increase in their performance post pandemic outbreak. 
Table 2: Results of Paired t-test

\begin{tabular}{|l|c|c|c|}
\hline Indices & P-Value & t- value & Decision $\mathbf{H}_{\mathbf{0}} \mathbf{\text { }}$ \\
\hline 1. S\&P BSE Healthcare Index & 0.844 & 0.20 & Accepted \\
\hline 2. Sun Pharma & 0.000 & 8.33 & Rejected \\
\hline 3. Dr. Reddy's Laboratories Ltd. & 0.007 & -2.85 & Rejected \\
\hline 4. Divi's Laboratories Ltd. & 0.000 & -8.97 & Rejected \\
\hline 5. Cipla Ltd./ India & 0.000 & 12.83 & Rejected \\
\hline 6. Piramal Enterprises Ltd. & 0.000 & 4.76 & Rejected \\
\hline 7. Apollo Hospitals Enterprise Ltd. & 0.001 & -3.61 & Rejected \\
\hline 8. Lupin Ltd. & 0.000 & 15.18 & Rejected \\
\hline 9. Aurobindo Pharma Ltd. & 0.211 & -1.28 & Accepted \\
\hline 10. Biocon Ltd. & 0.160 & -1.44 & Accepted \\
\hline 11. Torrent Pharmaceuticals Ltd. & 0.000 & -6.40 & Rejected \\
\hline
\end{tabular}

To test whether there is any significant difference in the market behavior of S\&P BSE Healthcare Index and its top ten constituents pre and post pandemic outbreak, paired t-test was conducted and Table 2 highlights the results. As we can see in three cases (i.e., in S\&P BSE Healthcare index, Aurobindo pharma Ltd. \& Biocon Ltd.), the null hypothesis is being accepted which means the pandemic outbreak has had made no difference in their stock price behavior. It remained, more or less, same after the pandemic outbreak and we found no improvement or deterioration. In relation to the remaining eight sample Pharma companies, we rejected the null hypothesis. This means that outbreak of the pandemic has had made a significant difference in the market behavior of their stock prices. As per the value of the $t$ statistic, the difference in the stock prices of the sample companies are both positive and negative. While the stock price of some companies soared up after the pandemic, the stock price of some companies have also plunged during the said period.

\section{CONCLUSION}

The pandemic outbreak has caused a widespread chaos and the stock market is not also free from its clutches. While the performance of other sectoral indices downgraded due to travel restrictions, market closures, lower Foreign Direct Investments, disruptions in supply chain and transportation logistics due to curfews, etc., the pharma sector is the least affected one. Amid the widespread turmoil, the pharma sector has outperformed both in BSE and NSE stock exchanges. The pharma sector and healthcare companies may be benefitted by the COVID-19 outbreak by supplying necessary medications to the US and other foreign countries. S\&P BSE Healthcare Index despite of the widespread disturbances caused by the pandemic outbreak showed a surge in its performance post pandemic outbreak. Moreover, among its top ten constituents, the performance of most of the companies went up post the pandemic outbreak.
As per the t-statistic conducted in the present study, S\&P BSE Healthcare Index do not show any deviation in its market behavior, i.e., there had been no increment or decrement in its performance due to the pandemic outbreak. While among its top ten constituent companies, eight companies (Sun Pharma, Dr. Reddy's Laboratories Ltd., Divi's Laboratories Ltd., Cipla Ltd./ India, Piramal Enterprises Ltd., Apollo Hospitals Enterprises Ltd., Lupin Ltd., and Torrent Pharmaceuticals Ltd.) showed deviation in their market behavior post pandemic outbreak, i.e., there was either an increase or decrease in their respective performance. On the other hand, only two companies (Aurobindo Pharma Ltd. \& Biocon Ltd.) do not show any deviation in their market behavior post the pandemic outbreak. So on a common ground, we can say that most of the pharma sector companies have witnessed deviations or difference in their market behavior post the pandemic outbreak.

The present study is not also free from limitations. Firstly, for the study only a short duration of time, i.e., 50 days pre and post pandemic outbreak has been taken. Secondly, out of the S\&P BSE Healthcare Index, the market behavior of only top ten constituent companies have been taken into consideration.

\section{REFERENCES}

1. Meltzer, M. I., Cox, N. J., Fukuda, K. (1999). The Economic Impact of Pandemic Influenza in the United States: Priorities for Intervention. Emerging Infectious Diseases. 5(5), 659-671.

2. Brainerd, E., \& Siegler, M. V. (2003). The Economic Effects of the 1918 Influenza Epidemic. 3791, C.E.P.R. Discussion Papers.

3. Overby, J., Rayburn, M., \& Hammond, K. (2004). The China syndrome: The impact of the SARS epidemic in Southeast Asia. Asia Pacific Journal of Marketing and Logistics, 16(1), 69-94

4. Bloom, E., de Wit, V., \& Carangal-San Jose M. J. (2005). Potential economic impact of an avian flu pandemic on Asia. Manila, Philippines: Asian Development Bank.

5. Burns, A., Mensbrugghe, D., \& Hans, T. (2006). Evaluating the economic consequences of Avian Influenza. Global Development Finance. 


\section{Retrieved}

from

http://www.worldbank.org/gdf2006.

6. Garrett, T. A. (2007). Economic Effects of the 1918 Influenza Epidemic.

7. Enemark, C. (2009). Is Pandemic Flu a Security Threat? Survival, 51(1), 191-214. doi:10.1080/00396330902749798

8. Doshi, P. (2011). The elusive definition of pandemic influenza. Bulletin of the World Health Organization, $\quad$ 89(7), 532-538. doi:10.2471/blt.11.086173

9. Sahu, M., Singh, N., Shukla, M. K., Potdar, V. A., Sharma, R. K., Sahare, L. K., Ukey, M. J., \& Barde, P. V. (2017). Molecular and epidemiological analysis of pandemic and postpandemic influenza A(H1N1)pdm09 virus from central India. Journal of Medical Virology, 90(3), 447-455. doi:10.1002/jmv.24982

10. Holmberg, M., \& Lundgren, B. (2018). Framing post-pandemic preparedness: Comparing eight European plans, Global Public Health, 13(1), 99114, doi: 10.1080/17441692.2016.1149202

11. Bloom, D. E., Cadarette, D., \& Sevilla, J. (2018). New and resurgent infectious diseases can have far-reaching economic repercussions. Finance and Development, 55(2), 46-49.

12. Fan V. Y., Jamison, D. T., \& Summers, L. H. (2018). Pandemic risk: how large are the expected losses? Bulletin of the World Health Organization, 2018(96), 129-134. doi: http://dx.doi.org/10.2471/BLT.17.199588

13. Kostava, D., Cassell, C.H., Rodd, J. T., Williams, D. E., Singh, T., Martel, L. D., \& Bunnell, R. E. (2019). Long distance effects of epidemics: Assessing the link between the 2014 West Africa Ebola outbreak and U.S. exports and employment. Wiley Health Economics. 28(11), 1248-1261. doi: 10.1002/hec.3938

14. Guimbeau, A., Menon, N., \& Musacchio, A. (2019). The Brazilian Bombshell? The short and long-term impact of the 1918 influenza pandemic the South American way.

15. Meng, L., Hua, F., \& Bian, Z. (2020). Coronavirus Disease 2019 (COVID-19): Emerging and Future Challenges for Dental and Oral Medicine. International \& American Association for Dental Research 2020: Journal of Dental Research, 00(0), 1-7.

16. Karlsson, M., Nilsson, T., \& Pichler, S. (2020). The Impact of the 1918 Spanish Flu Epidemic on Economic Performance in Sweden. Journal of Health Economics, 36, 1-19.

17. Delivorias, A., \& Scholz, N. (2020). Economic impact of epidemics and pandemics. EPRS European Parliamentary Research Service, 1-10

18. Rajagopal, D. (2020, March 13). Covid-19 Impact: Fears of Chinese API shortage hurt pharma players. The Economic Times. Retrieved from

https://economictimes.indiatimes.com/markets/st ocks/news/covid-19-impact-fears-of-chinese-apishortage-hurt-pharmaplayers/articleshow/74606020.cms

19. Mahesh, P. (2020, March 23). For mutual fund investors, pharma is the new equity safe haven. The Economic Times. Retrieved from https://economictimes.indiatimes.com/mf/analysis /for-mf-investors-pharma-is-the-new-equity-safehaven/printarticle/74766417.cms

20. Somvanshi, K., K. (2020, February 26). Is specialty business 'a go or a no-go' segment for pharma companies? The Economic Times. Retrieved from https://economictimes.indiatimes.com/markets/st ocks/news/is-specialty-business-a-go-or-a-no-gosegment-for-pharmacompanies/articleshow/74312726.cms

21. Langlois, S. (2020, March 19). Market action a century ago suggests worst could be over for stocks, if not for the coronavirus pandemic. Market Watch. Retrieved from https://www.marketwatch.com/story/marketbehavior-a-century-ago-suggests-the-worstcould-be-over-for-stocks-if-not-for-thecoronavirus-pandemic-2020-03-19

22. McKibbin, W., \& Fernando, R. (2020, March 2). The Global Macroeconomic Impacts of COVID19: Seven Scenarios. Brookings. Retrieved from https://www.brookings.edu/research/the-globalmacroeconomic-impacts-of-covid-19-sevenscenarios/ 